\title{
The impact of microbubble-enhanced therapeutic ultrasound combined with prothrombin on microwave ablation in the rabbit liver
}

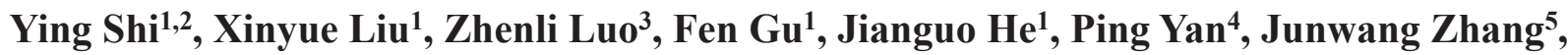 \\ Xiaodong Zhou ${ }^{1}$
}

${ }^{1}$ Department of Ultrasound, Xijing Hospital, Fourth Military Medical University, Xi'an, ${ }^{2}$ Department of Ultrasound, the Second Hospital of Shanxi Medical University, Taiyuan, ${ }^{3}$ Department of Cardiology, Xijing Hospital, Fourth Military Medical University, Xi'an, ${ }^{4}$ Department of Biochemistry and Molecular Biology, Shanxi Medical University, Taiyuan, ${ }^{5}$ Department of Gastroenterology, Xijing Hospital, Fourth Military Medical University, Xi'an, China.

\begin{abstract}
Aim: This study aimed to investigate the effect of microbubble-enhanced ultrasound (MEUS) combined with prothrombin on regional hepatic circulation and microwave ablation (MWA) in rabbit livers. Materials and methods: High-pressureamplitude therapeutic ultrasound (TUS) was used to treat 52 surgically exposed livers of healthy New Zealand rabbits: 13 livers were treated with MEUS alone, 13 with MEUS and prothrombin (PMEUS), 13 with ultrasound plus normal saline and 13 with ultrasound plus prothrombin as controls. Contrast-enhanced ultrasound (CEUS) imaging was performed on the exposed livers before and after treatment, and acoustic quantification was done to assess liver perfusion. Then, the liver was divided into two parts, one was used for pathologic examination and the other was ablated with microwave (MWA) and then processed for pathologic examination. Results: The CEUS images and Peak value after treatment in the PMEUS group were significantly reduced as compared to the remaining 3 groups $(\mathrm{p}<0.05)$. Occasional piecemeal hemorrhage was evidenced in the pathological examination in the MEUS group. Obvious cellular degeneration and necrosis with thrombosis were observed in the PMEUS group. Electron microscopy showed endothelial damage in both the MEUS group and PMEUS group. After MWA, coagulated volumes $(\mathrm{V})$ in the PMEUS group were larger than in the remaining 3 groups $(\mathrm{p}<0.05)$. The cell ultrastructure disorder was more severe in the PMEUS group than in remaining 3 groups. Conclusion: PMEUS promotes endothelial injury and produces more obvious thrombotic occlusion, improving the therapeutic effect of MWA on the rabbit liver.

Keywords: liver, microbubble-enhanced ultrasound, prothrombin, microwave ablation
\end{abstract}

\section{Introduction}

Several image-guided ablation techniques have been developed in order to treat patients with unresectable hepatocellular carcinoma (HCC). These minimally invasive procedures can achieve effective and reproducible

Received 06.04.2016 Accepted 14.07.2016

Med Ultrason

2016, Vol. 18, No 4, 438-445

Corresponding author: Xiaodong Zhou, MD, PhD, Department of Ultrasound, Xijing Hospital

Fourth Military Medical University

17 Changle Xilu,710032, Xi'an, China

Phone: +86-029-84775447

Fax: +86-029-84775447

E-mail: zhouxd1@sina.com tumor destruction with low morbidity. Percutaneous ablation is accepted as the best therapeutic choice for patients with early-stage HCC when resection or transplantation is not available [1,2]. Thermal ablation employs localized heating or freezing and enables in situ destruction of malignant liver tumors without damage to normal liver parenchyma. The thermal ablation in clinical practice includes hyperthermic treatments (radiofrequency [RF] ablation, microwave ablation [MWA] and laser ablation) and hepatic cryotherapy [3,4]. Minimally invasive, image-guided ablation has reduced cost and morbidity as compared to standard surgical resection, and can be used to treat patients who are not surgical candidates. However, thermal ablation has limitations: persistent growth of residual tumor at the ablation margin, inability to ef- 
fectively treat large tumors, and variability in complete treatment based on tumor location [5]. MWA is frequently used for the treatment of malignant liver tumors, being associated with few complications but is effective only for tumors $<3 \mathrm{~cm}$ in diameter [6].

In order to improve the therapeutic efficacy of thermal ablation studies on the blockage or reduction of liver blood flow, the procedures have been limited to the transcatheter embolization of hepatic arteries and surgical Pringle maneuver [7-9]. Therefore, less invasive techniques that may selectively obstruct regional liver blood flow are desirable.

Acoustic cavitation is one of the major physical effects of ultrasound (US). Inertial cavitation, whereby microbubbles serve as cavitation nuclei, is believed to be the major cause of these US bioeffects [10-12]. Ultrasound agent-induced endothelial damage can be inherently thrombogenic or aid sclerotherapeutic thrombogenesis through the application of subtherapeutic doses of thrombogenic drugs [13]. The measured inertial cavitation dose correlates with the degree of vascular endothelial injury in the presence of ultrasound contrast agent [14]. Regional liver blood perfusion can be temporarily blocked by microbubble-enhanced ultrasound (MEUS) with high-pressure amplitude [15].

Despite the vascular effects induced by high-pressure amplitude, MEUS can block blood perfusion in the rabbit spleen and prothrombin helps to enhance and extend these effects [16]. We hypothesized that the combination of MEUS and prothrombin would result in the ablation of a larger volume of liver tissues, possibly because the prothrombin induced circulation blockage promotes intravascular thrombosis. In this study, we aim to demonstrate that MEUS plus prothrombin has an enhanced capability to block local blood perfusion in the liver and this can increase the volume of liver ablated.

\section{Materials and methods}

\section{Animals}

Fifty two healthy New Zealand rabbits weighing 2.0-2.5 kg were purchased from the Laboratory Animal Center of the Fourth Military Medical University. All rabbits were randomly divided into four individual groups: Group A, sham therapeutic ultrasound exposure plus normal saline group (sham+NS, n=13); Group B, sham therapeutic ultrasound exposure plus normal saline plus prothrombin group (sham $+\mathrm{NS}+\mathrm{P}, \mathrm{n}=13$ ); Group C, therapeutic ultrasound plus microbubble group (MEUS, $\mathrm{n}=13$ ); Group D, therapeutic ultrasound plus microbubble plus prothrombin group (PMEUS, $n=13$ ). The experimental procedures in this study were approved by the Animal Care and Use Committee of the university (No: XJYYLL-2013016). The animals were killed in a humane manner by a prescribed method.

\section{Microbubbles}

The sonographic contrast agent SonoVue (Bracco SpA, Milan, Italy), including the active substance sulfur hexafluoride, was in the form of microbubbles. Other ingredients included macrogol 4000, distearoylphosphatidylcholine, dipalmitoylphosphatidylglycerol sodium, and palmitic acid- for microbubble formation. The average diameter of microbubbles was $2.5 \mu \mathrm{m}$. The microbubble suspension was prepared according to the manufacturer's instructions, and the final concentration of sulfur hexafluoride was $8 \mu \mathrm{L}(45 \mu \mathrm{g}) / \mathrm{mL}$. For the CEUS, a bolus of $0.02 \mathrm{~mL} / \mathrm{kg}$ microbubble suspension was injected via the ear vein. For the nucleation in MEUS treatment, SonoVue was constantly administered at the rate of approximately $0.5 \mathrm{~mL} / \mathrm{min}$ to a total dose of $0.2 \mathrm{~mL} / \mathrm{kg}$ (diluted in $5 \mathrm{~mL}$ of saline).

\section{Therapeutic ultrasound device}

The therapeutic ultrasound (TUS) transducer comprised an air-backed, spherically concave disk $(25 \mathrm{~mm}$ in diameter, Kunshan Risheng Electronics, Kunshan, China) with a $160-\mathrm{mm}$ radius of curvature. A wave generator and a specially designed power amplifier $(250-350 \mathrm{~V}$ peak-to-peak, Mianyang Sonic Electronics, Mianyang, China) drove the transducer. The transducer had an aluminum shell, and the front aperture (28 $\mathrm{mm}$ in diameter) of the shell was covered with a polyimide membrane. To provide acoustic coupling, the 10-mm-long front chamber of the disk was filled with degassed water. The geometrical focus of the transducer was exactly $150 \mathrm{~mm}$ from the tip, where the diameter of US beam was $28 \mathrm{~mm}$. A needle hydrophone (TNU001A, NTR, Seattle, WA, USA) adjusted by a precise 3-D motion stage was set up to measure the acoustic output of the transducer at a range of $1 \mathrm{~cm}$ outside the tip. The transducer was operated at $831 \mathrm{kHz}$ with a 400 -cycle pulse length and a pulse repetition frequency of $9 \mathrm{~Hz}$. The acoustic pressure (peak negative pressure) output was $4.6 \mathrm{MPa}$. The transducer worked in an intermittent mode of $6 \mathrm{~s}$ on and $6 \mathrm{~s}$ off. The intermittent mode was used for microbubble disruption and reperfusion [16-18]. The actual working duty cycle was approximately $0.22 \%$.

\section{Prothrombin}

Hemocoagulase Atrox for Injection [19] (Nuokang Bio-pharmaceutical Inc., Shandong, China), separated and purified by Bothrops atrox, was in the form of white powder. Other ingredients included mannitol, gelatin (hydrolysis) and calcium chloride. It has the hemostatic activity, but may not reduce thrombocytes and thus may no increase the risk for thrombosis. 


\section{Treatments}

A 21-gauge needle was inserted into the peripheral auricular vein to establish the intravenous access, and animals were further intravenously anesthetized with $2 \%$ pentobarbital at $1.5 \mathrm{~mL} / \mathrm{kg}$. The anesthetized animals were placed in a supine position and the hair on the upper abdomen was removed by using $8 \%$ sodium sulfide. Under anesthesia, a midline incision was made for laparotomy. The liver was surgically exposed and a portion of the left liver lobe was slightly pulled out. Then, the liver was fixed in situ with saline gauze around it.

The TUS transducer with coupling agent was placed on the liver with minimal pressure. The transducer covered a circular area of approximately $28 \mathrm{~mm}$ in diameter at the distal half of the lobe. TUS exposure lasted $10 \mathrm{~min}$, and intravenous injection of normal saline $(0.5 \mathrm{~mL} / \mathrm{min})$ was done simultaneously in Group A and B. Then $2 \mathrm{U} / \mathrm{mL}$ prothrombin in $1 \mathrm{~mL}$ of saline was injected into the target area in Group B. In Group C and Group D TUS treatment lasted $10 \mathrm{~min}$ and intravenous injection of microbubble solution $(0.5 \mathrm{~mL} / \mathrm{min})$ was simultaneously performed. Then $2 \mathrm{U} / \mathrm{mL}$ prothrombin was injected into the target

Table I. Animal group assignment.

\begin{tabular}{llll}
\hline Group & $\begin{array}{l}\text { Intravenous } \\
\text { injection } \\
\text { (mL/kg) }\end{array}$ & $\begin{array}{l}\text { TUS treatment } \\
(\mathbf{1 0} \text { min) }\end{array}$ & $\begin{array}{l}\text { Local injection } \\
\text { of prothrombin }\end{array}$ \\
\hline $\mathrm{A}$ & NS 0.2 & Sham TUS & 0 \\
$\mathrm{~B}$ & NS 0.2 & Sham TUS & $2 \mathrm{U} / \mathrm{ml}$ \\
$\mathrm{C}$ & microbubble 0.2 & TUS & 0 \\
$\mathrm{D}$ & microbubble 0.2 & TUS & $2 \mathrm{U} / \mathrm{ml}$ \\
\hline
\end{tabular}

TUS - therapeutic ultrasound

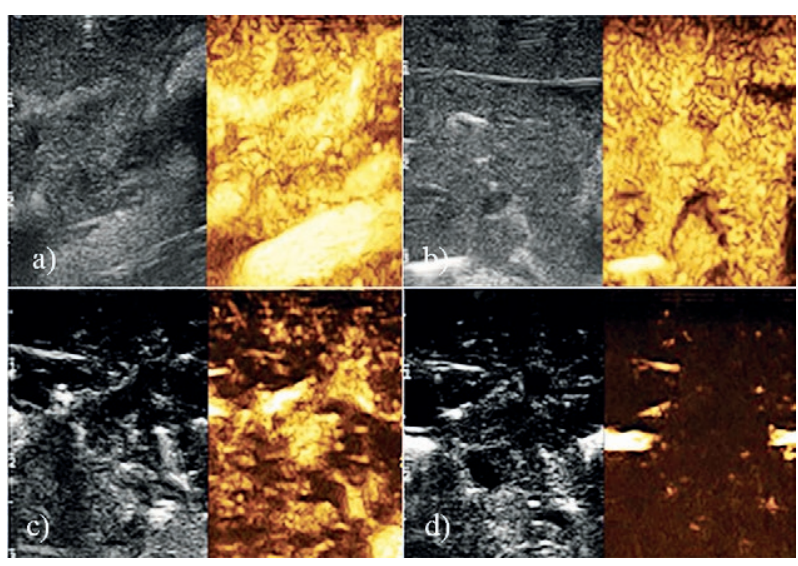

Fig 1. Two-dimensional contrast-enhanced ultrasound images at peak time after treatment: a) and b) The liver was well perfused at peak time after sham TUS treatment, and the liver filling remained unchanged after treatment in control groups; c) and d) Significant filling defect was observed at targeted regions in Group C and Group D at peak time after MEUS, and this was more obvious in Group D. area in Group D (Table I). The dose of microbubbles used for nucleating cavitation was $0.2 \mathrm{~mL} / \mathrm{kg}$.

During the procedure, CEUS imaging was performed on the exposed livers $10 \mathrm{~min}$ before (the baseline) and after treatment with a commercial diagnostic ultrasound system (Esaote EchoLaser equipped with a 9L highfrequency linear array probe, frequency range, 4.0-11.0 $\mathrm{MHz}$; Esaote Healthcare, Italy). The contrast imaging can detect contrast signal using a low mechanical index $(\mathrm{MI}=0.06)$ followed by an intravenous bolus injection of SonoVue lipid microbubbles at $0.02 \mathrm{~mL} / \mathrm{kg}$. The conditions for CEUS were identical in the whole study. All CEUS images were stored digitally for further analysis (fig 1).

After CEUS imaging, animals in each group were divided into two subgroups for histologic examination $(n=3)$ and assessment of liver MWA $(n=10)$, respectively. In brief, 3 animals were intravenously anesthetized with $120 \mathrm{mg}$ of pentobarbital immediately after CEUS. The treated liver lobes were harvested for pathological examination. For liver MWA, the left treated lobes of 10 animals received MWA with a microwave therapeutic system (ECO-100C, equipped with a cooled-shaft antenna of $3 \mathrm{~mm}$ in diameter, the microwave irradiation frequency is 2,450 MHz, Nanjing ECO Microwave System Co. Nanjing, China). The MWA was done at 10 Wfor 2 minbecause MWA at a higher power may cause larger coagulated volumes that exceed the thickness of liver lobes. All animals were intravenously anesthetized with $120 \mathrm{mg}$ of pentobarbital immediately after MWA, and the treated livers were collected for pathological examination.

\section{CEUS image analysis}

For CEUS, a region of interest (ROI) about $6 \times 6 \mathrm{~mm}$ was defined at the central treated region of the liver using the Qcontrast quantification software (Esaote Medical Systems) connected to a computer. ROI was defined at the same site in the middle part of the liver. The parameters reflecting hepatic blood perfusion, such as time to peak (TP) and peak echogenicity (Peak), were calculated automatically. TP refers to the time from the beginning of the rise in the CEUS curve to the end at the Peak. The Peak represents the peak intensity or peak acoustic intensity and is the average echogenicity within an ROI at the peak contrast enhancement.

\section{Pathological Examinations}

After MEUS (or sham TUS), the targeted livers of 3 rabbits in each group were collected and processed for electron microscopy and light microscopy (H\&E staining).

After MWA, the whole liver was collected and stored in phosphate-buffered saline (PBS) immediately. Coagulated liver tissues were cut into two parts along the direction of the needle. The maximum length (L, millimeters), 
width (W, millimeters), and depth (D, millimeters) of the coagulated region in the liver were measured, and the volume (V, cubic millimeters) was calculated according to the following formula [20]: $\mathrm{V}=\pi / 6 \times \mathrm{L} \times \mathrm{W} \times \mathrm{D}$. After ablation, the liver tissues were collected. The center piece with surrounding areas in each sample was divided in half and respectively processed for electron microscopy and light microscopy examination (H\&E staining).

\section{Statistical analysis}

All the coagulated liver volumes and the Peak in CEUS are expressed as means \pm standard deviations (SD). Comparisons of coagulated liver volume among 4 groups were done with one-way analysis of variance (ANOVA). The Peak was compared with one way ANOVA among groups and that obtained before and after treatment was compared with the paired $t$ test. A value of $p$ less than 0.05 was considered statistically significant. Statistical analysis was conducted using the SPSS version 13.0 (SPSS, Cary, NC, USA).

\section{Results}

\section{Visual analysis of CEUS}

Before treatment, livers in all groups showed rapid and complete filling of contrast agent and there was no filling defect. After treatment the liver filling remained unchanged in Group A and Group B as compared to that before treatment. In Group C and Group D, the liver showed uneven local perfusion with perfusion defect, which were more obvious in Group D (fig 2).

\section{Gray-scale analysis}

All 52 livers displayed a homogenous contrast enhancement before treatment $(\mathrm{F}=2.734, \mathrm{p}=054)$. After treatment there was a significant difference in the Peak before and after treatment in Group C and Group D ( $<<0.05)$; no significant difference in the Peak before and after treatment in Group A and Group B was found ( $>>0.05$ ). The Peak after treatment in Group D was significantly higher than that in the remaining 3 groups $(\mathrm{p}=0.0001)$. The Peak after treatment in Group $\mathrm{C}$ was significantly higher than that in Group A and Group B ( $\mathrm{p}=0.0001)$. There was no significant difference in the Peak between Group A and Group B ( $\mathrm{p}=0.069)$ (Table II).

\section{Macroscopic Findings}

After MWA, the targeted liver tissues in all groups were cut along the needle traces. These tissues were oval and dark yellow and included little carbon black area at the ablation center. A sharp boundary clearly separated the coagulated tissues from the surrounding tissues (fig 3).

The length, width, depth, and volume of the coagulated tissues were significantly different among the 4 groups

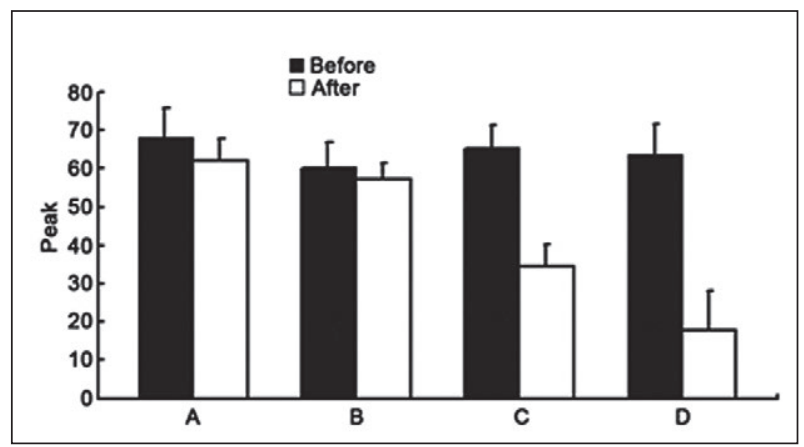

Fig 2. Gray-scale analysis. In two control groups, the Peak value remained homogenous after treatment. In MEUS and PMEUS groups, the Peak value reduced significantly after treatment. The Peak value in PMEUS group displayed a 2-fold reduction as compared to the MEUS group.

Table II. Peak intensity value before and after the treatment.

\begin{tabular}{llll}
\hline Group & Before treatment & After treatment & p \\
\hline A & $67.90 \pm 7.78$ & $62.16 \pm 5.63^{* \#}$ & 0.053 \\
B & $59.97 \pm 6.77$ & $57.23 \pm 4.17^{* \#}$ & 0.291 \\
C & $65.03 \pm 6.19$ & $34.35 \pm 5.97 \#$ & 0.000 \\
D & $63.58 \pm 7.88$ & $18.05 \pm 9.90^{*}$ & 0.000 \\
\hline
\end{tabular}

*Value is significantly different from the group $\mathrm{C}$ value, $\mathrm{p}<0.05$; \#Value is significantly different from the group $D$ value, $p<0.05$

Table III. Summary of morphologic examination of coagulated tissues by MWA in the 4 groups.

\begin{tabular}{lllll}
\hline Group & $\mathbf{L}(\mathbf{c m})$ & $\mathbf{W}(\mathbf{c m})$ & $\mathbf{D}(\mathbf{c m})$ & $\mathbf{V}\left(\mathbf{c m}^{3}\right)$ \\
\hline A & $1.23 \pm 0.06^{*} \#$ & $1.03 \pm 0.04^{*} \#$ & $0.84 \pm 0.13^{*} \#$ & $0.53 \pm 0.10^{* \#}$ \\
B & $1.23 \pm 0.15^{*} \#$ & $0.93 \pm 0.03^{* \#}$ & $0.81 \pm 0.05^{* \#}$ & $0.46 \pm 0.09^{* \#}$ \\
C & $1.58 \pm 0.14 \#$ & $1.43 \pm 0.11 \#$ & $1.47 \pm 0.28 \#$ & $1.65 \pm 0.51 \#$ \\
D & $1.96 \pm .094^{*}$ & $1.78 \pm 0.04^{*}$ & $1.79 \pm 0.37^{*}$ & $3.13 \pm 0.78^{*}$ \\
p value & 0.0001 & 0.0001 & 0.0001 & 0.0001 \\
\hline
\end{tabular}

$\mathrm{L}$ : the maximum length of the coagulated region; $\mathrm{W}$ : the maximum width of the coagulated region; D: the maximum depth of the coagulated region; V: the volume of the coagulated region.* Value is significantly different from the group $\mathrm{C}$ value; \# Value is significantly different from the group $\mathrm{D}$ value.

$(\mathrm{p}=0.0001)$. The maximum length, width, and depth and the mean volume of the coagulated tissues after MWA in Group D were significantly greater than those in the remaining 3 groups $(p=0.0001)$. The maximum length, width, and depth and the mean volume of the coagulated tissue after MWA in Group C were markedly greater than those in Group A and Group B ( $p=0.0001)$. The maximum length, width, depth, and volume were comparable between Group A and Group B (Table III).

\section{Light microscopy}

The livers were harvested immediately after CEUS and subjected to H\&E staining. Results showed the untreated liver lobes had normal architecture. Normal 


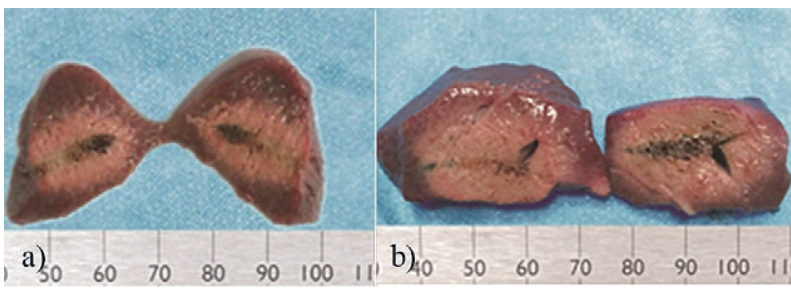

Fig 3. The targeted regions of rabbit livers treated by MWA. The targeted regions of rabbit livers treated by MWA were dark yellow. The coagulated volume in Group D (b) was larger than in Group C (a).

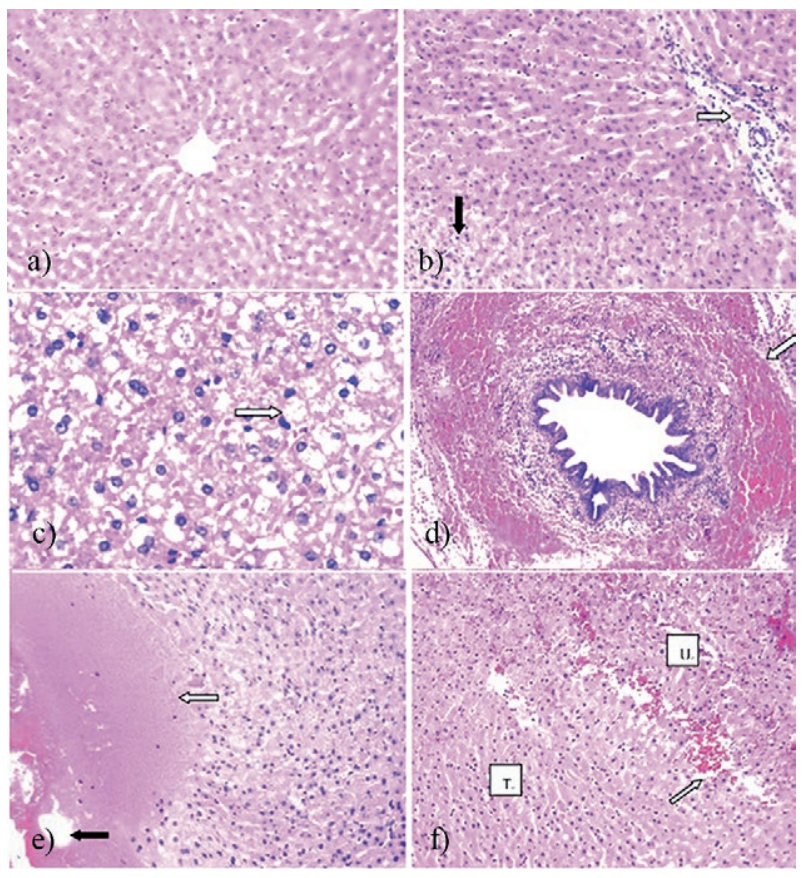

Fig 4. Hematoxylin and eosin staining of the liver in different groups: a) Sham TUS treated liver in control groups had hepatocytes with normal architecture and observable sinusoids; b) After microbubble-enhanced ultrasound treatment in MEUS group, there was obvious hepatocyte swelling, the sinusoids became squeezed and shrunken (black arrow), and there were focal, small flake hemorrhages (white arrow); c) and d) After PMEUS in Group D, there was obvious hepatocyte swelling, sinusoids were squeezed and shrunken (c, white arrow), and sleeve-like hemorrhage was also found at the portal areas (d, white arrow); e) After MWA, there was a cavity (black arrow) in the gasification area and the carbide area had no cellular structure and was homogeneously red (white arrow); f) A clear demarcation border was present between targeted tissues (T) and untreated tissues (U). There were a lot of "ghost RBC" which had no hemoglobin in the hepatic sinus and blood vessels in the untreated tissues (white arrow). Bars $=50 \mu \mathrm{m}(\mathrm{a}, \mathrm{b}$, d-f) and $100 \mu \mathrm{m}(\mathrm{c})$. a, b, d, e, f: $\times 100$; $: \times 400$. hepatocytes were regularly arranged with visible sinusoids in Group A and Group B (fig 4a). However, after MEUS, hemorrhage was found in the portal area and there was hepatocyte swelling in Group C and Group D. In Group C, there were a small amount of flake hemorrhage and hepatocyte swelling after treatment (fig $4 \mathrm{~b}$ ); in Group D, there were visible hepatocyte swelling (fig 4c) and diffused hemorrhage at the portal area, usually the sleeve-like hemorrhage (fig 4d) after treatment.

After MWA, a cavity was present in the central gasification area around which was the carbide area with no basic cellular structure and being homogeneously red (fig 4e). The border between the surrounding tissues and the targeted tissues was clear in 4 groups because there were a lot of "ghost red blood cells (RBC)" which had no

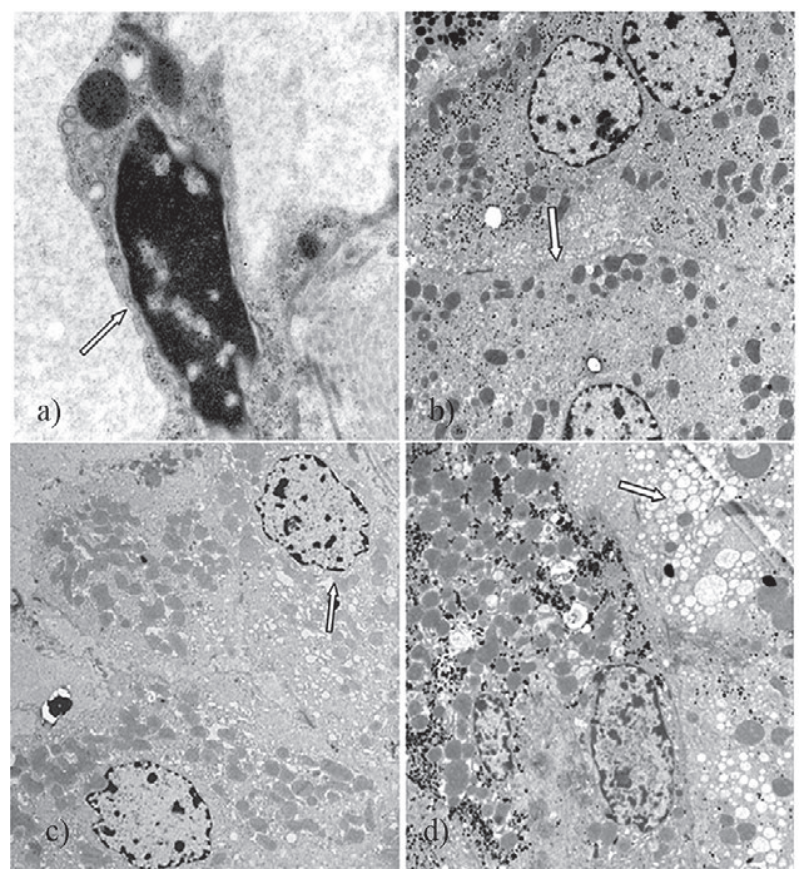

Fig 5. Ultrastructure of the liver in different groups (Electron microscopy): a) In vascular endothelial cells (white arrow), heterochromatin was increased and damaged, and these cells protruded towards the luminal side of the portal area in the MEUS group and PMEUS group (Scale bar: $0.5 \mu \mathrm{m}$ ); b) The structure of the targeted liver tissue and the hepatocyte membrane in control groups were observable, and the nuclear membrane (white arrow) was intact (Scale bar: $5 \mu \mathrm{m}$ ); c) The hepatic sinusoid interspace and cell junctions were significantly destroyed, and cell karyotypes were irregular, and there was a broadening gap (white arrow) around the nucleus in the PMEUS group (Scale bar: $2 \mu \mathrm{m}$ ); d) In the border between targeted tissues and surrounding tissues, the swelling hepatocytes exhibited typical ballooning degeneration, in which a large number of bubbles (white arrow) were found in the PMEUS group (Scale bar: 2 $\mu \mathrm{m})$. 
hemoglobin in the hepatic sinus and no blood vessels in the untreated tissues (fig 4f). However, the morphologic characteristics of the coagulated tissues were similar among the 4 groups. In the ablation areas, hepatic plates and hepatic cords were observable, and the basic outline of the hepatocytes and cell nuclei appeared normal.

\section{Electron microscopy}

After MEUS (or sham TUS) the livers were harvested immediately for electron microscopy. Results showed the heterochromatin in vascular endothelial cells was increased and damaged, and these cells protruded to the luminal side in the portal area of Group C and Group D (fig $5 a)$. However, the endothelial cells in the hepatic sinus in Group A and Group B were intact. After MWA most of the hepatic sinusoid interspaces, cell junctions and cell membranes in the targeted regions were intact in Groups A and Group B (fig 5b). However, in Group C and Group $\mathrm{D}$, the hepatic sinusoid interspaces and cell junctions were significantly destroyed, and cell karyotypes were irregular with widening perinuclear space. These changes were more obvious in Group D (fig 5c). At the border between targeted tissues and surrounding tissues the swelling hepatocytes exhibited typical ballooning degeneration, in which a large number of bubbles were found in Group C and Group D (fig 5d).

\section{Discussions}

Our results showed the combination of MEUS and prothrombin considerably improved the circulation blockage and ablative ability in the normal rabbit liver. PMEUS combined techniques resulted in an average 6-fold enhancement in ablation volume over control groups, about 2-fold enhancement over MEUS and about 2 -fold reduction in the Peak over MEUS. Therefore, the volume of ablation correlates with hepatic blood perfusion. In addition, electron microscopy showed that PMEUS resulted in increased cell coagulation necrosis by MWA via reducing the heat sink effect.

It has been shown that bubble-based ultrasound contrast agents may provide nuclei for inertial cavitation in the rotating tube exposure system [10]. Miller et al [11] for the first time showed that the diagnostic ultrasound activation of contrast agent gas bodies was able to induce capillary rupture in mice. Several previous studies have also shown that microbublble enhanced ultrasound with high-pressure amplitude can induce vascular wall damage in single small vessels, which is proportional to the peak negative pressure amplitude of the insonifying pulses. The mechanical effect of inertial cavitation is believed to be a major cause of endothelial damage $[13,14,21]$. Gao et al [15] found that the liver circula- tion could be temporally "shut down" for 15-30 min by extracorporeal MEUS. However, MEUS is difficult to produce a complete thrombotic occlusion without intravascular thrombin. Liu et al [16] for the first time found that the combination of MEUS and prothrombin could enhance and prolong the vascular effects of MEUS for up to $1 \mathrm{~h}$, and the peak intensity dropped from $20.2 \pm 2.70$ $\mathrm{dB}$ to $11.6 \pm 4.58 \mathrm{~dB}$ immediately after treatment. This was confirmed in this study. In the presence of SonoVue microbubbles and TUS (or sham TUS) at a frequency of $831 \mathrm{KHz}$ with the peak negative of $4.6 \mathrm{MPa}$, our results showed the regional Peak in experimental groups (Group C and D) dropped significantly, and the circulation achieved a 2-fold increase after PMEUS as compared to MEUS alone). In control groups (Group A and B) there was no ultrasonic cavitation, and thus obvious regional microvascular blocking and pathological changes were not observed after experiment. In Group $\mathrm{C}$ MEUS produced regional microvascular blocking and corresponding pathological changes in a few vessels, but the regional microvascular damage in Group D was more obvious and mainly ascribed to the thrombosis and cell degeneration necrosis. This related to the action of prothrombin, because prothrombin, Hemocoagulase Atrox for Injection, is an enzymatic hemostatic with high purity and can be activated by the batroxobin and blood coagulation factorX. Prothrombin has shown favorable safety and effectiveness [19], and may not produce blood coagulation in the normal vascular system. Thus, in control group (sham exposure plus NS) thrombin at the same dose did not result in intravascular thrombosis. Its pharmacological effects after MEUS caused regional microvascular damage in the PMEUS group.

Hwang et al [13] found that additional thrombin accelerated the coagulation cascade, resulting in immediate intravascular thrombosis. Liu et al [16] also found that prothrombin could help to enhance and extend the vascular effects. The pathological changes in the liver of this study also confirmed the above findings. Electron microscopy showed obvious vascular endothelial injury in the MEUS and PMEUS groups, and light microscopy revealed, in the PMEUS group, thrombosis and cell degeneration were more common as compared to the MEUS group. Our study showed that MEUS could induce vascular endothelial injury and mild thrombosis, and PMEUS could further promote the vascular effects, resulting in diffused thrombosis.

In the past years, some studies have indicated that microbubbles in experimental animals can increase the volume of coagulated tissues, thus improving the therapeutic efficacy. With microbubble enhanced HIFU ablation, Luo et al [22] found $0.2 \mathrm{~mL}$ of SonoVue increased the 
coagulation volume by 3.0 folds after a 2 -second HIFU ablation. In the study of Liu et al [18] MEUS combined with ethanol ablation was used, achieving a 10 times increase in the volume as compared to that ablated by ethanol injection only. These studies confirm that MEUS can increase the liver ablation volume. In this study, cessation of the hepatic circulation provides an opportunity to greatly increase the disruption capability of MWA. After MEUS, targeted liver tissues were ablated with a microwave at $10 \mathrm{~W}$ for $2 \mathrm{~min}$, and the volume of ablated tissues was 4 times larger than that in control group. After PMEUS, the volume of ablated tissues was 6 times larger than that in the control group. This indicates that PMEUS and MEUS can significantly reduce the heat sink effect, enhance the volume of coagulation, and increase the ablation volume. Thus, this technique might be clinically applicable in large liver cancers.

Our study showed there was no diffuse necrosis in the ablation area in 4 groups under a light microscope and the hepatocyte structure remained visible. This may be related to the use of low power microwave (10 W, $2 \mathrm{~min})$ in this study. However, the electron microscopy revealed that the ultrastructure of the cells in all the groups was destroyed to a various extent. More injured nuclear membranes were found in the MEUS group and the PMEUS group suggesting that the tissues are more severely damaged in the presence of MEUS, especially a large number of damaged nuclei were found in the PMEUS group. Bubbles were detectable in all the groups. However, more bubbles appeared in the PMEUS group under electron microscopy, which might be related to the more severe damage to the cell ultrastructure. The high temperature caused by MWA at the ablation regions results in the denaturation of proteins and other cellular constituents as well as thermal fixation, and thus the tissues in the treated area are resistant to degradation.

There were still limitations in our study. We did not observe the long-term effects of this technique on the liver. This technique was not employed in an in vivo tumor model. Thus, whether this technique is effective in the treatment of liver cancer, especially the large liver cancer, is required to be further studied. In addition, the relationships among acoustic parameters, the vascular effects and ablation volume remain unknown.

\section{Conclusion}

MEUS combined with prothrombin may promote the MEUS induced endothelial damage and produce a larger thrombotic area, resulting in a significant reduction in the heat sink effect and enhancement in the efficacy of MWA in rabbit livers.

\section{Acknowledgements}

We thank Wen Luo and Lu Han for their assistance in the animal experiments. This work was supported by the National Natural Science Foundation of China grant 81371567.

\section{Conflict of interest: none}

\section{References}

1. Llovet JM, Di Bisceglie AM, Bruix J, et al. Design and endpoints of clinical trials in hepatocellular carcinoma. J Natl Cancer Inst 2008;100:698-711.

2. Bruix J, Sherman M; American Association for the Study of Liver Diseases. Management of hepatocellular carcinoma: an update. Hepatology 2011;53:1020-1022.

3. Lencioni R, Crocetti L. Image-guided ablation for hepatocellular carcinoma. Recent Results Cancer Res 2013;190:181-194.

4. Dick EA, Taylor-Robinson SD, Thomas HC, Gedroyc WM. Ablative therapy for liver tumours. Gut 2002;50:733-739.

5. Ahmed M, Goldberg SN. Basic science research in thermal ablation. Surg Oncol Clin N Am 2011;20:237-258.

6. Ng KK, Lam CM, Poon RT, Ai V, Tso WK, Fan ST. Thermal ablative therapy for malignant liver tumors: a critical appraisal. J Gastroenterol Hepatol 2003;18:616-629.

7. Chinn SB, Lee FT Jr, Kennedy GD, et al. Effect of vascular occlusion on radiofrequency ablation of the liver: results in a porcine model. AJR Am J Roentgenol 2001;176:789-795.

8. Chang CK, Hendy MP, Smith JM, Recht MH, Welling RE. Radiofrequency ablation of the porcine liver with complete hepatic vascular occlusion. Ann Surg Oncol 2002;9:594-598.

9. Burdio F, Mulier S, Navarro A, et al. Influence of approach on outcome in radiofrequency ablation of liver tumors. Surg Oncol 2008;17:295-299.

10. Miller DL, Thomas RM. Ultrasound contrast agents nucleate inertial cavitation in vitro. Ultrasound Med Biol 1995;21:1059-1065.

11. Miller DL, Quddus J. Diagnostic ultrasound activation of contrast agent gas bodies induces capillary rupture in mice. Proc Natl Acad Sci U S A 2000;97:10179-10184.

12. Maruvada S, Hynynen K. Optical monitoring of ultrasound-induced bioeffects in glass catfish. Ultrasound Med Biol 2004;30:67-74.

13. Hwang JH, Brayman AA, Reidy MA, Matula TJ, Kimmey MB, Crum LA. Vascular effects induced by combined $1-\mathrm{MHz}$ ultrasound and microbubble contrast agent treatments in vivo. Ultrasound Med Biol 2005;31:553-564.

14. Hwang JH, Tu J, Brayman AA, Matula TJ, Crum LA. Correlation between inertial cavitation dose and endothelial cell damage in vivo. Ultrasound Med Biol 2006;32:16111619.

15. Gao Y, Gao S, Zhao B, et al. Vascular effects of microbubble-enhanced, pulsed, focused ultrasound on liver blood perfusion. Ultrasound Med Biol 2012;38:91-98. 
16. Liu J, Xiao S, Wu S, et al. Disruption of splenic circulation using microbubble-enhanced ultrasound and prothrombin: a preliminary study. Ultrasound Med Biol 2012;38:1930-1937.

17. Liu Z, Gao S, Zhao Y, et al. Disruption of tumor neovasculature by microbubble enhanced ultrasound: a potential new physical therapy of anti-angiogenesis. Ultrasound Med Biol 2012;38:253-261.

18. Liu Q, Zhao H, Wu S, et al. Impact of microbubble-enhanced ultrasound on liver ethanol ablation. Ultrasound Med Biol 2013;39:1039-1046.

19. Wan SY, Hu YC, Zhan YQ, Qin DD, Ding Y. Hemocoagulase atrox reduces vascular modeling in rabbit carotid artery adventitia. Int J Clin Exp Pathol 2013;6:2386-2395.
20. Di Matteo F, Martino M, Rea R, et al. US-guided application of Nd:YAG laser in porcine pancreatic tissue: an ex vivo study and numerical simulation. Gastrointest Endosc 2013;78:750-755.

21. Samuel S, Cooper MA, Bull JL, Fowlkes JB, Miller DL. An ex vivo study of the correlation between acoustic emission and microvascular damage. Ultrasound Med Biol 2009;35:1574-1586.

22. Luo W, Zhou X, Ren X, Zheng M, Zhang J, He G. Enhancing effects of SonoVue, a microbubble sonographic contrast agent, on high-intensity focused ultrasound ablation in rabbit livers in vivo. J Ultrasound Med 2007;26:469476. 